\title{
An in vitro assessment by means of laser Doppler velocimetry of the Medtronic Advantage bileaflet mechanical heart valve hinge flow
}

Rahul Saxena, MS ${ }^{\mathrm{a}}$

Jack Lemmon, PhD ${ }^{\mathrm{a}}$

Jeffrey Ellis, $\mathrm{PhD}^{\mathrm{b}}$

Ajit Yoganathan, $\mathrm{PhD}^{\mathrm{a}}$

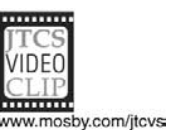

Objective: The aim of this study was to evaluate the hinge flow field characteristics of the Medtronic Advantage bileaflet valve and compare them with the flow fields of the St Jude Medical standard valve. The present study provides laser Doppler velocimetry results for the Advantage and St Jude Medical valves to make a direct comparison of the flow fields of the 2 valve designs. This study aids in determining the preclinical efficacy of Medtronic's new bileaflet valve hinge design.

Methods: Two-dimensional laser Doppler velocimetry was used to measure the velocities in the hinge regions of size 29 Medtronic Advantage and St Jude Medical standard bileaflet valve designs. Exact dimensional models of the bileaflet valves, including the hinge regions, were cast from transparent plastic materials to conduct these measurements under simulated physiologic conditions. Laser Doppler velocimetry measurements were conducted under physiologic conditions, with the valves placed in the mitral position of a pulsatile flow loop. Measurements were taken at several elevation levels in the hinge region. Multiple measurement locations obtained in each plane provided a grid work by which the flow fields could be detailed.

Results: Velocity measurements obtained for each valve design in the hinge recess were used to reconstruct the flow fields. For the Advantage valve, the peak velocities during leakage flow in the hinge at zero depth, one-third depth, and two-thirds depth levels into the hinge recess were $0.9,1.6$, and $1.8 \mathrm{~m} / \mathrm{s}$, respectively. Corresponding values for the St Jude Medical valve were 1.3, 1.6, and $2.1 \mathrm{~m} / \mathrm{s}$, respectively. From the reconstructed flow fields, the flow patterns seen within the 2 hinge designs exhibited similar features, with more dynamic flow patterns observed in the Advantage hinge during the forward flow phase.

Biomedical Engineering ${ }^{\mathrm{a}}$ and the School of Mechanical Engineering, ${ }^{\mathrm{b}}$ Cardiovascular Fluid Mechanics Laboratory, Georgia Institute of Technology, Atlanta, Ga.

Supported by a research grant from Medtronic Heart Valves Division, Minneapolis, Minn.

Received for publication Sept 3, 2002; revisions requested Oct 1, 2002; revisions received Jan 14, 2003; accepted for publication Feb 11, 2003.

Address for reprints: Ajit P. Yoganathan, $\mathrm{PhD}$, Associate Chair, School of Biomedical Engineering, Georgia Institute of Technology, 315 Ferst Dr, IBB Building, Rm 1126, Atlanta, GA 30332-0535 (E-mail: ajit.yoganathan@bme.gatech.edu).

J Thorac Cardiovasc Surg 2003;126:90-8

Copyright $\odot 2003$ by The American Association for Thoracic Surgery

$0022-5223 / 2003 \$ 30.00+0$

doi:10.1016/S0022-5223(03)00581-6

Conclusions: The present study demonstrated that the hinge flow dynamics of the Advantage bileaflet design were similar to those of the St Jude Medical hinge design. The velocities within the hinge were slightly higher for the St Jude Medical valve but not significantly different. There appears to be more dynamic flow through the hinge of the Advantage valve during the forward flow phase.

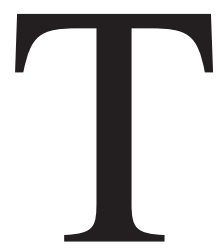

he bileaflet valve design is the most widely implanted mechanical heart valve prosthesis. Characteristics of the bileaflet valve include favorable forward flow hemodynamics, ease of implantation, durability, and successful clinical performance. The most clinically successful of the bileaflet designs has been the St Jude Medical (SJM) valves (St Jude Medical, Inc, St Paul, Minn), with 25 years of proved clinical performance. The SJM valves have presented very low long-term rates of thrombogenicity and valve-related complications. In contrast to the SJM valve, the Medtronic Parallel valve design (Medtronic, Inc, Minneapolis, Minn) exhibited 


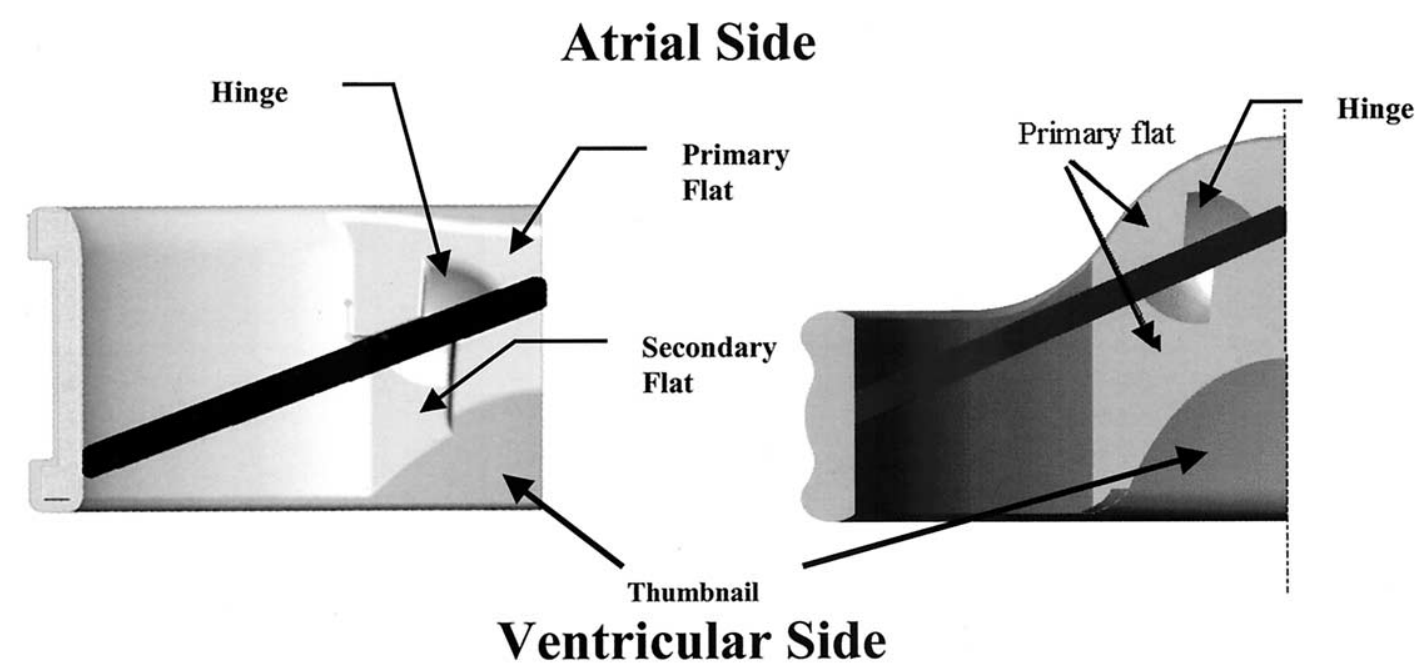

A

B

Figure 1. Schematics of the Advantage (A) and SJM (B) valve hinge designs with appropriate nomenclature.

unacceptably high thrombogenic rates during its clinical trial. In-depth studies have shown that the flow fields within the hinge mechanism are critical to the proper function of the bileaflet valve. ${ }^{1-6}$ Previous studies have detailed the detrimental effect on valve operation that the existing stagnation zones can have in developing thrombosis sites within the hinge area. Numerous studies have shown differences in the hinge flow patterns between several valve designs, and correlations between these results and clinical performance have been inferred. ${ }^{1,2,4,5}$ In the past, development of new valve designs lacked a priori studies of flow patterns in the hinge mechanism because of technical constraints with either experimental or computational methods. Technology is now available, both experimentally and computationally, that can meet the requirements of complex analysis of flow fields in the small recessed regions of mechanical bileaflet valves. These techniques have been used previously to study valve designs already in clinical use. ${ }^{1,7-11}$

This article presents the preclinical evaluation of a new bileaflet valve design from the Medtronic Heart Valves Division, with comparison of results with those of the proved SJM design. The Medtronic Advantage mechanical bileaflet valve is designed with a cylindrical housing that allows the leaflets to seat wholly within the housing when closed and incorporates a butterfly hinge design similar to that of the SJM valve, with a unique bilevel flat design feature (Figure 1). The Medtronic valve hinge design incorporates a secondary flat on the ventricular side of the butterfly hinge to promote improved flow washing characteristics through the hinge during the forward and leakage flow phases. The secondary flat is designed to decrease the resistance that flow encounters into and out of the hinge during the cardiac cycle by channeling the flow to and from the hinge recess. Laser Doppler velocimetry (LDV) was used to acquire the flow fields within the hinge region of the Medtronic and SJM bileaflet valves. The LDV investigation of the flow fields and direct comparison of results associated with the hinge region allows for evaluation of the Advantage design features. The present study presents LDV results in the hinge region of the SJM and Advantage valves at 3 elevations within the hinge to provide direct comparison of the flow fields of the 2 valve designs. This comparison aids in determining the preclinical efficacy of the new hinge design.

\section{Methods}

This study examined the flow characteristics of the Advantage and SJM standard bileaflet heart valves within the hinge regions of the valves by using LDV. Previous studies have shown that the flow patterns among the Standard, HP, and Regent valves are similar., Clear housing models were used to gain optical access to the hinge region of the models to obtain the velocity measurements in the small hinge recess. Experiments were performed on size 29 valve models in the mitral position under a single simulated cardiac condition. Description of the experimental apparatus, flow conditions, measurement techniques, and data analysis follows.

\section{Experimental Conditions}

Optical access into the hinge mechanism was obtained by using Medtronic's proprietary process to cast housings with a clear epoxy resin. These casts were made from clinical quality Advantage and SJM size 29 valves. This process reproduced accurate internal geometries of each valve, which included the internal orifice diameter, flat regions, hinge recesses, and housing shape. This casting provided an exact representation of the internal features of the clinical valve housings, and the clinical quality valve's pyrolytic carbon leaflets were fitted in the clear housing models to create exact replicas of the clinical valves. 

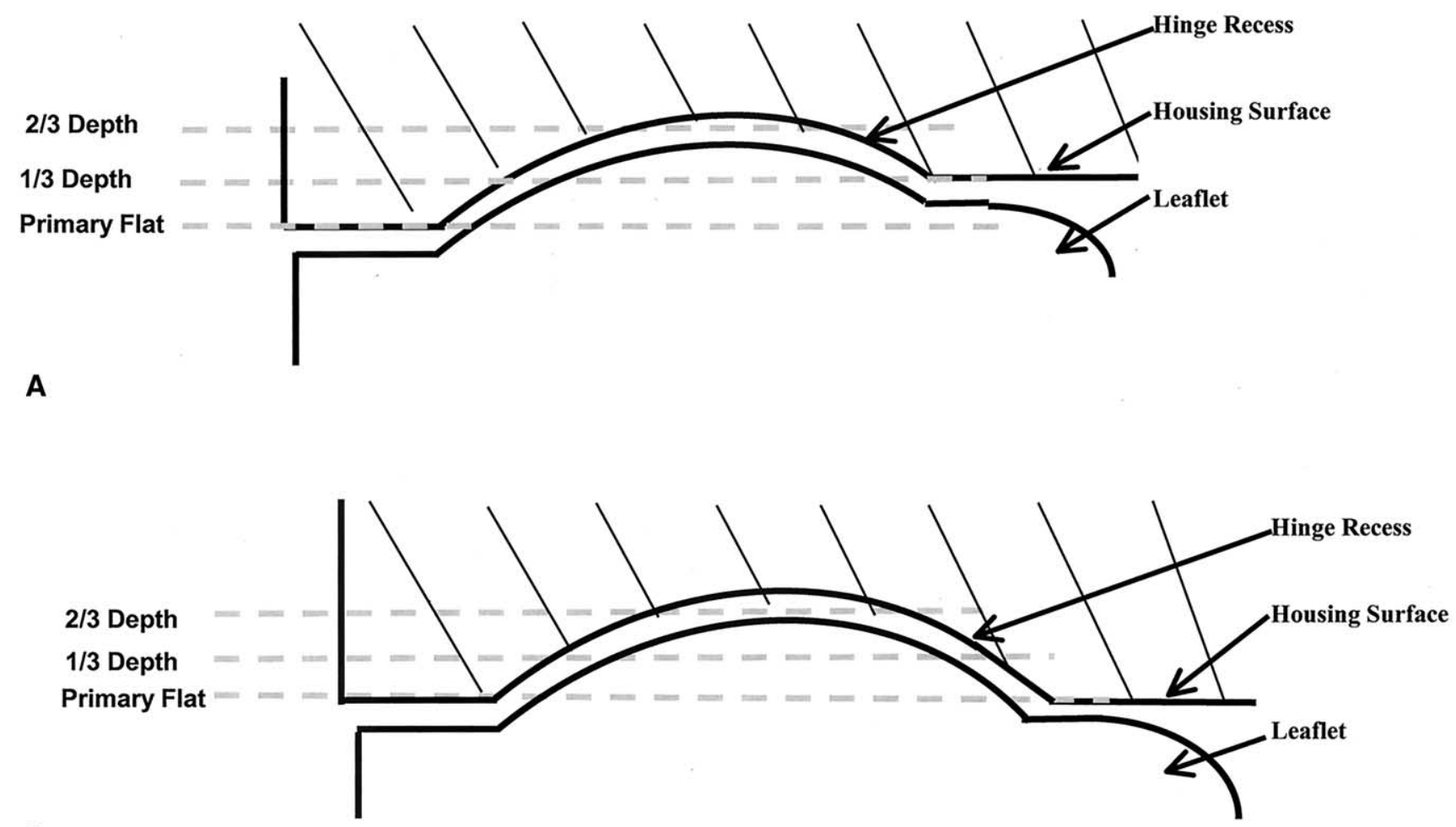

B

Figure 2. Measurement planes with nomenclature for the Medtronic Advantage (A) and SJM (B) hinge designs.

The valve models were mounted in the mitral position of the Georgia Tech left heart pulse duplicator, which has been previously described. ${ }^{4,5}$ With this system, physiologic mitral conditions (ie, heart rate of 70 beats/min [corresponding to an 860-ms cardiac cycle time], cardiac output of $4 \mathrm{~L} / \mathrm{min}$, peak diastolic flow rate of $12 \mathrm{~L} / \mathrm{min}$, diastolic duration of between 500 and $550 \mathrm{~ms}$, and peak left ventricular pressure of $160 \mathrm{~mm} \mathrm{Hg}$ ) were simulated for evaluating the flow through the valves. Flow rates were measured with an ultrasonic flowmeter (model T108/24N, Transonic Systems, Inc, Ithaca, NY), and pressures were measured with an Argon disposable pressure transducer (model 500503A, Athens, Tex).

The working fluid for the flow loop was a solution of $79 \%$ saturated aqueous sodium iodide, $20 \%$ glycerin, and $1 \%$ water by volume. This fluid had a kinematic viscosity of 3.5 centistokes to match that of blood at physiologic shear rates. The fluid refractive index was adjusted to match that of the clear valve housing, thereby minimizing optical distortion effects. The refractive index of each model was determined by measuring the travel of the laser over a known distance through the clear model. Silicone carbide particles (TSI Inc, Shoreview, Minn) with a nominal diameter of $1.5 \mu \mathrm{m}$ were used to seed the flow.

\section{Data Acquisition}

A fiberoptic, 3-component, coincident LDV system (Aerometrics Inc, Sunnyvale, Calif) was used to obtain 2-component velocity measurements within the hinge region of the Advantage and SJM clear-housing valve models. A 2-component fiberoptic transceiver probe with a $100-\mathrm{mm}$ focal-length lens was coupled to the fiber drive optics train to produce an ellipsoidal probe volume with minor and major axes of approximately $21 \mu \mathrm{m} \times 140 \mu \mathrm{m}$, respectively. All measurements were conducted in the backscatter mode. $^{4}$

Doppler signals measured in the flow field were processed with Fast Fourier Transform-based real-time signal analyzers. A commercial software package (Dataview v.0.084, Aerometrics) was used to acquire data and to control both the signal analyzers and the photomultiplier hardware. A resettable clock and a 3-channel analog-digital converter was interfaced to the pulse duplicator system to allow the laser Doppler velocity measurements to be synchronized with the mitral flow and left ventricular pressure waveforms.

\section{Measurement Sites}

The LDV measurements were performed at multiple elevations for the Medtronic Advantage valve and SJM valve models. At each level, the Doppler probe volume was traversed across the area of interest in a simple $\mathrm{x}-\mathrm{y}$ grid pattern to interrogate the region of interest. Three similar planes were investigated within the hinge to compare results between the Advantage and SJM valve hinge flow patterns (Figure 2). For this article, the plane level with the primary flat is presented for comparison of the flow fields between the 2 valve designs during forward and leakage flow. In addition, the velocity fields at 2 planes within the hinge are presented at peak systole to demonstrate the flow patterns within the recess of each valve during leakage flow. 


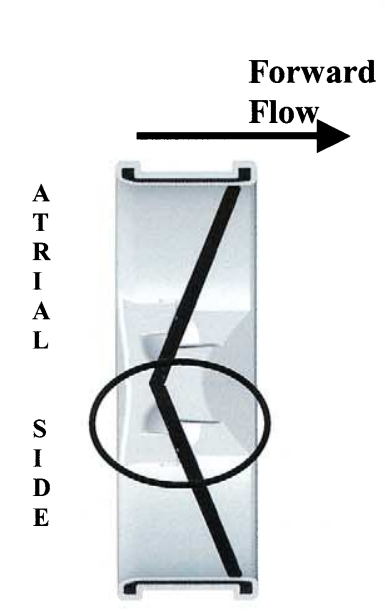

A

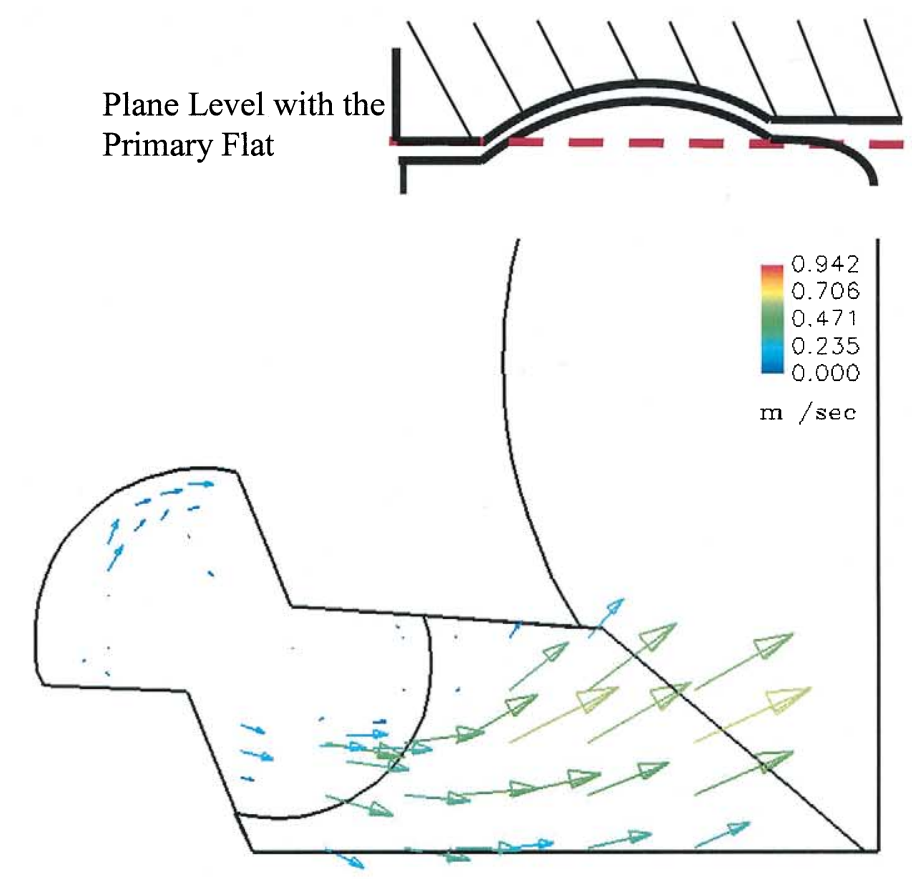

B

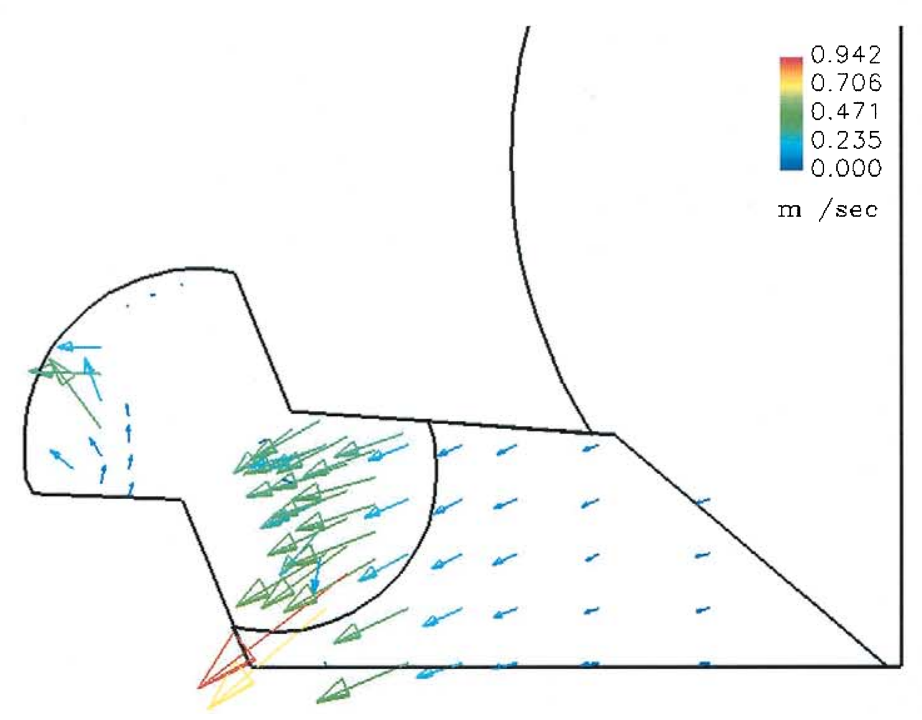

Figure 3. Velocity field at peak diastole (A) and peak systole (B) in the plane level with the primary flat at zero depth into the hinge for the Medtronic Advantage valve. The arrows point in the direction of mean velocity vectors and are color coded by velocity magnitude (in meters per second), as shown in the legend.

\section{Data Reduction}

The data acquired at each measurement site was divided into 20-ms time bins to decompose the pulsatile flow data into a series of discrete time intervals. A simple decomposition technique, which separated the mean flow velocity from the fluctuating velocities within each time bin, was used to obtain the mean velocities. ${ }^{12}$

\section{Results}

LDV measurements obtained in multiple planes (Figure 2) of each valve model were evaluated and compared. The results presented in Figures 3 to 6 are the mean velocity vectors within that plane at a particular time of the cardiac cycle. The vector length and color coding are representative 


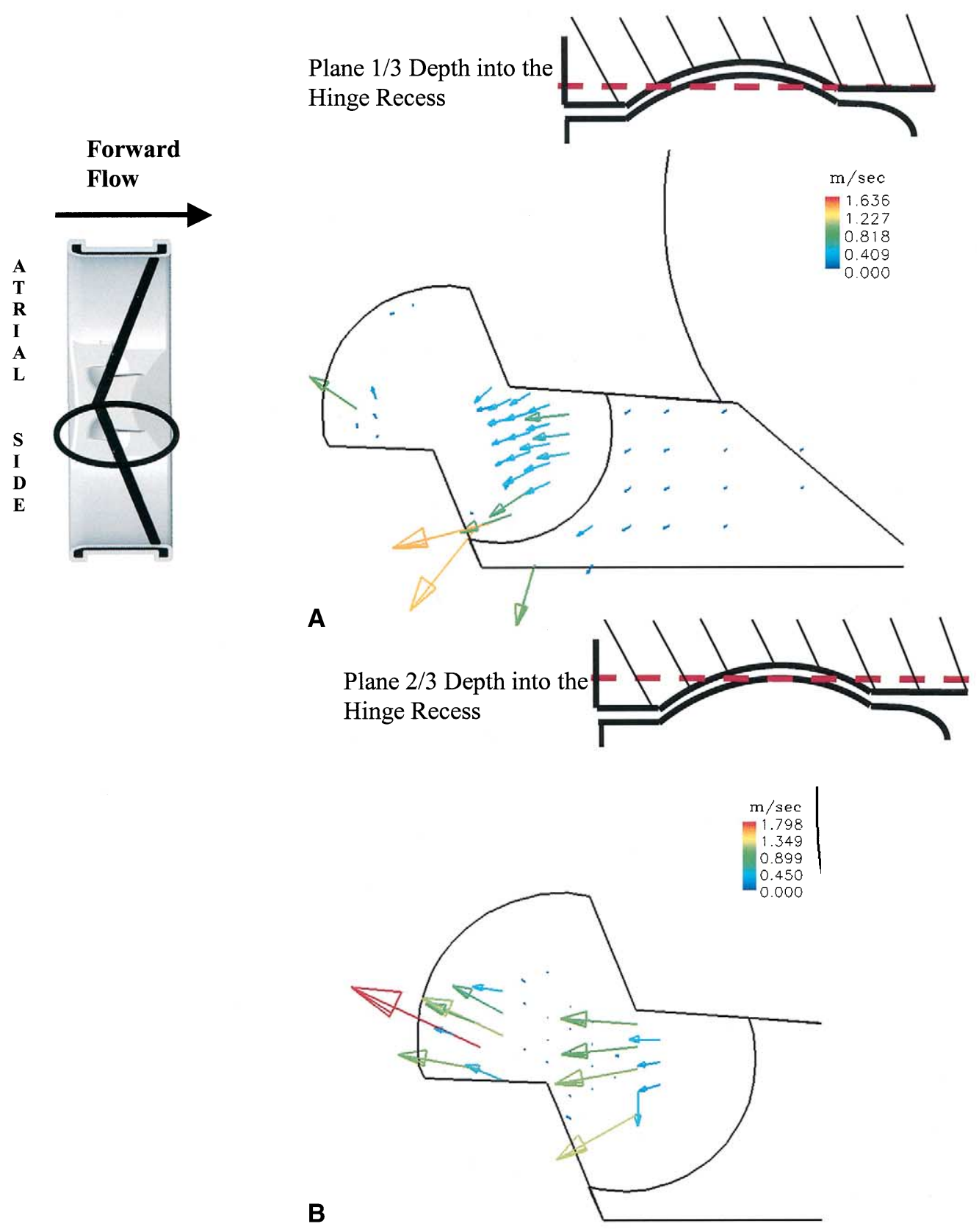

Figure 4. Velocity fields of the Advantage valve at one-third (A) and two-thirds (B) depth into the hinge recess, exhibiting similar flow patterns at the 2 levels with maximum velocities of 1.64 and $1.80 \mathrm{~m} / \mathrm{s}$, respectively.

of the velocity magnitude (in meters per second), as outlined by the legend in each figure.

\section{Medtronic Advantage: Level With the Primary Flat} This level (Figure 3) provided information on the flow entering and exiting the hinge recess. At this level, during forward flow, the highest velocities within the hinge were on the order of 0.30 to $0.50 \mathrm{~m} / \mathrm{s}$ at peak diastole (Figure 3, A). Flow on the atrial side of the hinge appeared to follow the perimeter of the hinge, whereas flow at the outlet was more axially oriented into the secondary flat, and the flow was then seen to accelerate and move toward the center of the valve. Velocities on the order of $0.50 \mathrm{~m} / \mathrm{s}$ were present below the secondary flat near the thumbnail. During the transition from mid-diastole to mid-deceleration, reverse flow was predominantly axial as it entered the secondary flat from the thumbnail. The flow then curved almost completely radially at the hinge-secondary flat interface, where 


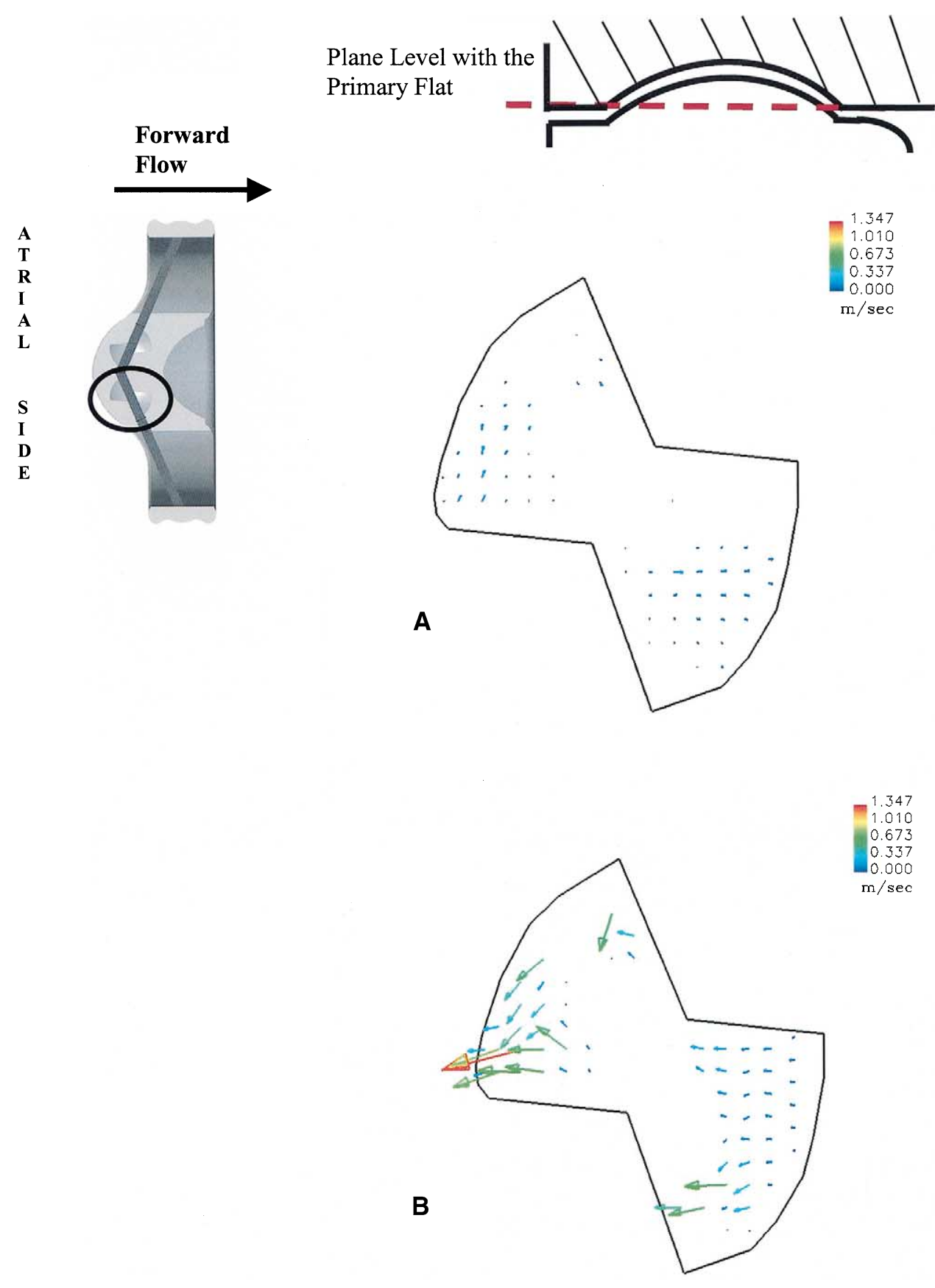

Figure 5. Velocity field at peak diastole (A) and peak systole (B) in the plane level with the primary flat at zero depth into the hinge of the SJM valve. The arrows point in the direction of mean velocity vectors and are color coded by velocity magnitude (in meters per second), as shown in the legend.

it moved away from the center of the valve. Reverse flow with velocities on the order of $0.20 \mathrm{~m} / \mathrm{s}$ can be seen to enter the hinge, moving radially away from the center of the valve. A uniform velocity field exits the lower corner of the hinge, while some recirculation can be observed in the upper corner of the hinge on the atrial side. During early systole, more uniform velocity fields with higher velocities on the order of 0.30 to $050 \mathrm{~m} / \mathrm{s}$ were observed entering the hinge. The velocities exiting the hinge on the atrial side were on the order of 0.10 to $0.35 \mathrm{~m} / \mathrm{s}$. The maximum observed velocity during leakage flow was less than $1 \mathrm{~m} / \mathrm{s}$. At late systole (Figure 3, B), the velocity field appeared uniform, entering the hinge with a magnitude of approximately $0.40 \mathrm{~m} / \mathrm{s}$. 

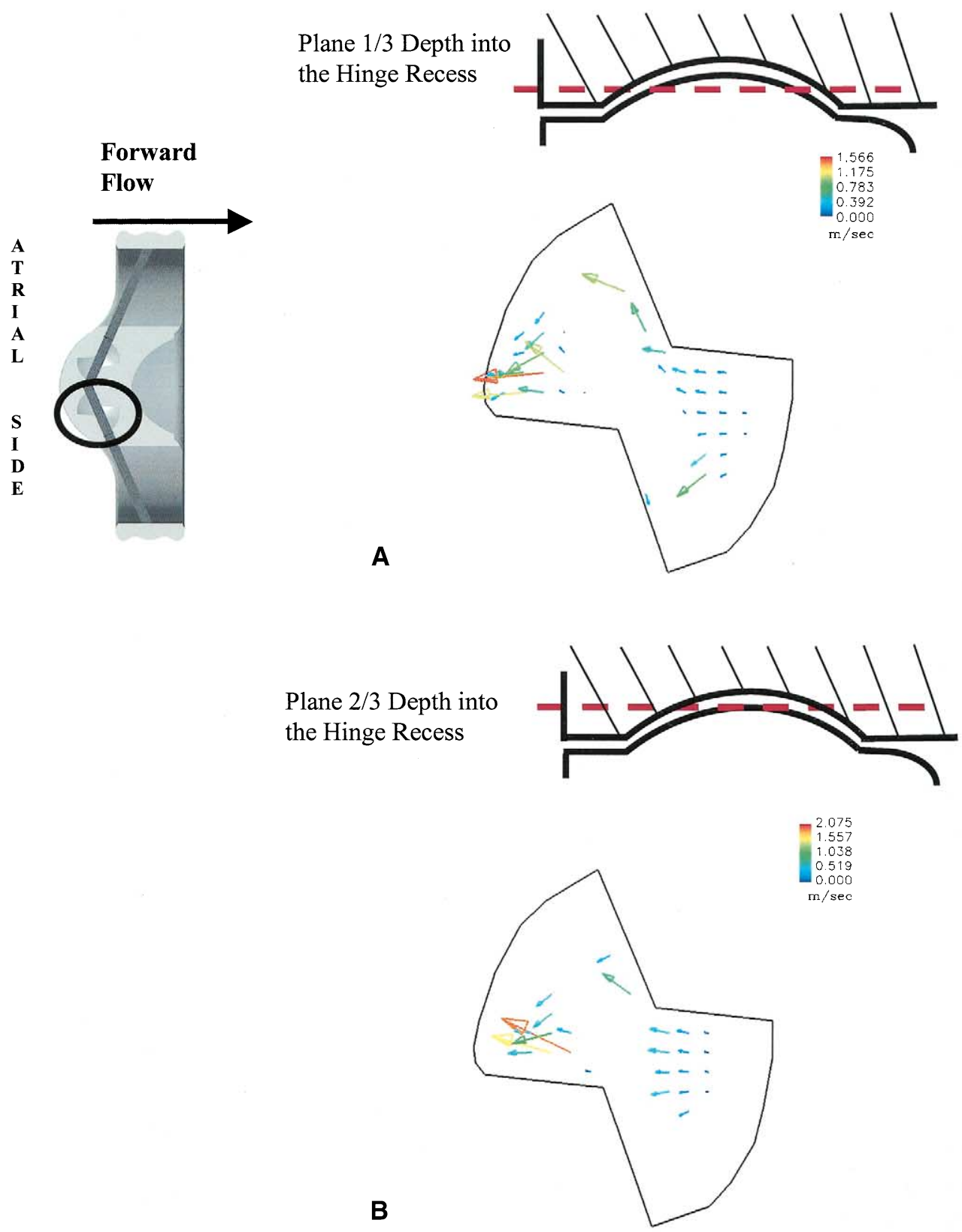

Figure 6. Velocity fields of the SJM valve at one-third (A) and two-thirds (B) depth into the hinge recess, showing similar flow patterns for the 2 planes with maximum velocities of 1.57 and $2.08 \mathrm{~m} / \mathrm{s}$, respectively.

\section{Medtronic Advantage: One-Third and Two-Thirds Depth Into the Hinge}

The velocity fields at one-third and two-thirds depth into the hinge recess during peak leakage flow were investigated and are shown in Figure 4. The flow entered the hinge on the ventricular side, with velocities of approximately 0.50 to $0.60 \mathrm{~m} / \mathrm{s}$ at the one-third depth into the hinge and 0.70 to $0.80 \mathrm{~m} / \mathrm{s}$ at the two-thirds depth into the hinge. On the atrial side, the flow was oriented axially out of the hinge, with peak velocities of 0.80 and $1.7 \mathrm{~m} / \mathrm{s}$ at the one-third and two-thirds depths, respectively. For the one-third depth, the maximum velocity of $1.6 \mathrm{~m} / \mathrm{s}$ was observed on the ventricular side of the hinge and could be due to the secondary flat-hinge transition under the leaflet.

\section{SJM: Level With the Primary Flat}

During forward flow, the highest velocities within the hinge were on the order of 0.10 to $0.30 \mathrm{~m} / \mathrm{s}$ between mid-acceleration and peak diastole (Figure 5, A). Flow at the hinge 
inlet appeared to follow the geometry of the hinge, whereas flow at the outlet was oriented in the axial direction. During early systole (Figure $5, B$ ), a predominantly axial velocity field with higher velocities on the order of 0.15 to $0.30 \mathrm{~m} / \mathrm{s}$ was observed entering the hinge. The velocities exiting the hinge are on the order of 0.50 to $0.80 \mathrm{~m} / \mathrm{s}$. Velocities on the atrial side of the hinge appeared to converge into the lower corner of the hinge. The lower corner of the hinge on the atrial side contained high velocities that reached $1.35 \mathrm{~m} / \mathrm{s}$ during the peak leakage flow phase of the cycle. During late systole, the velocity field was uniform, entering the hinge with magnitudes of approximately 0.10 to $0.20 \mathrm{~m} / \mathrm{s}$, and higher velocities on the order of $0.50 \mathrm{~m} / \mathrm{s}$ were measured on the atrial side.

\section{SJM: One-Third and Two-Thirds Depth Into the Hinge}

The velocity fields at one-third and two-thirds depth into the hinge recess during peak leakage flow are shown in Figure 6 . The flow entered the hinge uniformly on the ventricular side, with velocities of between 0.50 and $0.70 \mathrm{~m} / \mathrm{s}$ at the one-third and two-thirds depths, respectively. On the atrial side, the flow was directed toward the lower corner of the hinge, with peak velocities of approximately 1.5 and $2.0 \mathrm{~m} / \mathrm{s}$ at the one-third and two-thirds depths, respectively. The peak velocities are observed in similar locations on the atrial side at these 2 levels.

\section{Discussion}

Ellis and colleagues ${ }^{4,7}$ conducted LDV studies on SJM clear-housing valve models furnished by St Jude Medical that outlined the flow fields of the SJM valve design and related the flow characteristics to possible reasons for the valve's success in vivo. These results were reproduced for the current valve study to adjust for differences in experimental setup and flow waveform. The previous study ${ }^{4}$ also detailed the leaflet-wiping action of the SJM hinge, which created a washout pattern that restricted the persistence of separated and stagnation zones. The action created a doorslamming effect that in the in vivo case might serve to keep the hinge region cleared of any deposited and activated blood elements. The active leaflet motion through the SJM hinge is thus believed to be one of the contributing factors to the valve's successful clinical performance. Comparing the results from the Advantage and SJM measurements that show similar flow patterns and velocity magnitudes at the multiple planes illustrates that the Advantage valve has similar flow and leaflet dynamics as the SJM valve. Table 1 summarizes the peak velocities observed for each plane and valve design during the leakage and forward flow phases of the cardiac cycle.

The Medtronic Advantage valve design incorporates the same dynamics of the SJM valve's butterfly hinge design with one design difference. The presence of a secondary
Table 1. Peak measured leakage flow velocities within the hinge at each plane investigated

\begin{tabular}{lcc}
\hline Measurement plane & $\begin{array}{c}\text { Advantage maximum } \\
\text { leakage velocity }(\mathrm{m} / \mathbf{s})\end{array}$ & $\begin{array}{c}\text { SJM maximum } \\
\text { leakage velocity } \\
(\mathrm{m} / \mathbf{s})\end{array}$ \\
\hline $\begin{array}{l}\text { Level with primary flat } \\
\text { One-third depth into } \\
\text { hinge }\end{array}$ & 0.94 & 1.35 \\
$\begin{array}{l}\text { Two-thirds depth into } \\
\text { hinge }\end{array}$ & 1.64 & 1.57 \\
\hline
\end{tabular}

channel at the outflow of the butterfly hinge allows flow to enter and leave the hinge with less resistance than the flow through the SJM hinge. The effect of the secondary flat can be seen especially during the forward flow phase when comparing the Advantage and SJM velocity fields at the level of the primary flat (Figures 3 and 5). The highest velocities during diastole were around $0.50 \mathrm{~m} / \mathrm{s}$ for the Advantage design compared with approximately 0.20 to $0.30 \mathrm{~m} / \mathrm{s}$ for the SJM valve. During systole, the flow patterns within the Advantage hinge are more dynamic compared with those in the SJM valve. The flow dynamics during both systole and diastole result in more uniform flow through the hinge of the Advantage valve during forward and leakage flow, thereby reducing the potential for stagnation zones to be present inside the hinge.

The influence of the outflow channel can also be observed during the leakage flow of the cardiac cycle. As seen in Figures 3 and 5, the flow patterns at the level of the primary flat for the Advantage and SJM designs are similar. Velocities on the order of 0.15 to $0.30 \mathrm{~m} / \mathrm{s}$ enter the SJM hinge on the ventricular side, whereas the velocities typically ranged from 0.30 to $0.50 \mathrm{~m} / \mathrm{s}$ for the Advantage valve design. In comparison, the maximum velocity magnitude within the hinge was slightly lower for the Advantage design during the leakage flow. More importantly, the Advantage flow field appears to be more dynamic, but the full effect of this dynamic hinge washing cannot be determined without clinical results to evaluate the Advantage valve's performance.

As the levels progress further into the hinge recess, similarities continue to exist between the Advantage and SJM valve flow fields. Because of the similar butterfly hinge design, the Advantage valve appears to have comparable washing with that of the SJM hinge, with the velocities either equal or slightly lower for the Advantage valve at these levels within the hinge. These results provide assurance that the Advantage valve should have adequate washing of its hinge mechanism during the cardiac cycle. These observations are supported by the similarities between the Advantage and SJM hinge flow characteristics made in this direct comparison. 


\section{Experimental Limitations}

The LDV measurements were conducted only at selected locations and thus do not completely define the flow fields within the hinge region of the valves. Also, the measurements were acquired in a 2-dimensional plane, and any out-of-plane velocities (ie, change in the orientation of the flow) are not represented. It is possible that these confined flow fields might indeed be 3-dimensional because of the complex geometries formed by the interaction of the leaflet and hinge. Other experimental techniques, such as highspeed flow visualization or the use of scaled-up models, might be useful in assessing the flow fields more completely. The primary experimental limitations that prevented more detailed interrogations were the optical access of the models and the width of the gap between each leaflet and housing, leading to low data rates in some regions. Another limitation of the present study is that of the use of rigid mounted models. However, the present simplified setup helps isolate various factors that might contribute to the influence of the hinge flow dynamics and provides a way to make direct comparison of different valve designs.

\section{Conclusions}

A 2-dimensional LDV study was conducted on the Medtronic Advantage and SJM mechanical (size 29) heart valve designs under simulated physiologic flow conditions. This study provided detailed information on the hinge flow characteristics of the Advantage valve design compared with the SJM design to assess preclinical design efficacy by means of direct comparison of flow fields for the 2 designs. The Advantage design was found to have good washout characteristics during the forward and leakage flow phases of the cardiac cycle. The flow patterns within the hinge were observed to be similar to those of the SJM valve, but more dynamic flow patterns were observed in the hinge during forward flow when the valve was open. The Advantage's hinge flow might be enhanced by a secondary flat on the ventricular side of the hinge that appears to channel flow into and out of the hinge with less resistance than the SJM valve. Direct comparisons of results between the Advantage and SJM valve models show that velocity and flow patterns were comparable between the 2 valve designs. On the basis of the similar flow patterns and velocity magnitudes observed between the 2 valve designs, the Advantage valve should have adequate washing during operation, but true performance will be measured from the clinical outcomes.

\section{References}

1. Gross JM, Shu MC, Dai FF, Ellis JT, Yoganathan AP. A microstructural flow analysis within a bileaflet mechanical heart valve hinge. J Heart Valve Dis. 1996;5:581-90.

2. Ellis JT, Healy TM, Fontaine AA, Saxena R, Yoganathan AP. Velocity measurements and flow patterns within the hinge region of a Medtronic Parallel bileaflet mechanical valve with clear housing. J Heart Valve Dis. 1996;5:591-9.

3. Healy TM, Fontaine AA, Ellis JT, Walton S, Yoganathan AP. Visualization of the hinge flow in a 5:1 scaled model of the Medtronic Parallel bileaflet heart valve prosthesis. Exp Fluids. 1998;25:512-8.

4. Ellis JT, Yoganathan AP. A comparison of the hinge and near-hinge flow fields of the St Jude Medical Hemodynamic Plus and Regent bileaflet mechanical heart valves. J Thorac Cardiovasc Surg. 2000; 119:83-93.

5. Leo H, He Z, Ellis JT, Yoganathan AP. Microflow fields in the hinge region of the Carbomedics bileaflet mechanical heart valve design. J Thorac Cardiovasc Surg. 2002;124:561-74.

6. Keggen LA, Black MM, Lawford PV, Hose DR, Strachan JR. The use of enzyme activated milk for in vitro simulation of prosthetic valve thrombosis. J Heart Valve Dis. 1996;5:74-83.

7. Ellis JT, Travis BR, Yoganathan AP. An in vitro study of the hinge and near-field forward flow dynamics of the St. Jude Medical Regent bileaflet mechanical heart valve. Ann Biomed Eng. 2000;28:524-32.

8. Gao ZB, Hosein N, Dai FF, Hwang NH. Pressure and flow fields in the hinge region of bileaflet mechanical heart valves. J Heart Valve Dis. 1999;8:197-205

9. Wang J, Yao H, Lim CJ, Zhao Y, Yeo TJH, Hwang NHC. Computational fluid dynamics study of a protruded hinge bileaflet mechanical heart valve. J Heart Valve Dis. 2001;10:254-63.

10. Kelly SGD, Verdonck PR, Vierendeels JAM, Riemslagh K, Dick E, Van Nooten GG. A three-dimensional analysis of flow in the pivot regions of an ATS bileaflet valve. Int J Artif Organs. 1999;22:754-63.

11. Subramanian A, Mu H, Kadambi JR, Wernet MP, Brendzel AM, Harasaki H. Particle image velocimetry investigation of intravalvular flow fields of a bileaflet mechanical heart valve in a pulsatile flow. J Heart Valve Dis. 2000;9:721-31.

12. Baldwin JT, Deutsch S, Geselowitz DB, Tarbell JM. Estimation of Reynolds stresses within the Penn State left ventricular assist device. ASAIO Trans. 1990;36:M274-8. 